\title{
Efficient Structured Language Modeling for Speech Recognition
}

\author{
Ariya Rastrow, Mark Dredze, Sanjeev Khudanpur \\ Center for Language and Speech Processing, and \\ Human Language Technology Center of Excellence, Johns Hopkins University \\ \{ariya, mdredze, khudanpur\}@jhu.edu
}

\begin{abstract}
The structured language model (SLM) of [1] was one of the first to successfully integrate syntactic structure into language models. We extend the SLM framework in two new directions. First, we propose a new syntactic hierarchical interpolation that improves over previous approaches. Second, we develop a general information-theoretic algorithm for pruning the underlying Jelinek-Mercer interpolated LM used in [1], which substantially reduces the size of the LM, enabling us to train on large data. When combined with hill-climbing [2] the SLM is an accurate model, space-efficient and fast for rescoring large speech lattices. Experimental results on broadcast news demonstrate that the SLM outperforms a large 4-gram LM.
\end{abstract}

\section{Introduction}

Automatic Speech Recognition (ASR) relies on a language model (LM) to provide a strong linguistic prior on word sequences. Most language models rely on simple $n$-gram statistics and a wide range of smoothing and backoff techniques. Despite being simple and efficient, it is widely believed that limiting the context to only the $(n-1)$ most recent words ignores the structure of language, and several statistical frameworks have been proposed to incorporate the "syntactic structure of language back into language modeling" [1,3,4]. The Structured Language Model (SLM) [1] was one of the first successful attempts to build a statistical language model based on syntactic information. The SLM assigns a joint probability $P(W, T)$ to every word sequence $W$ and every possible binary parse tree $T$, where $T$ 's terminals are words $W$ with part-of-speech (POS) tags, and its internal nodes comprise non-terminal labels and lexical "heads" of phrases. Other approaches include using the exposed headwords in a maximum-entropy based LM [5], using exposed headwords from full-sentence parse tree in a neural network based LM [3], and the use of syntactic features in discriminative training [6]. However, most previous SLMs are large, complex and impractical for real-life applications.

We propose a new SLM based on dependency structures derived using a state-of-the-art probabilistic shift-reduce parser [7], in contrast to the original SLM which parametrizes the parser component with a conditional model. Two key improvements are also made to the SLM. First, we propose a simple hierarchical interpolation of syntactic parameters that achieves better performance without significant model complexity. Second, we maintain model efficiency on a large training corpus by using a general information-theoretic pruning method to reduce the size of the underlying Jelinek-Mercer LM [8]. When combined with fast hill-climbing rescoring [6], our framework is accurate, space efficient and practical for ASR.

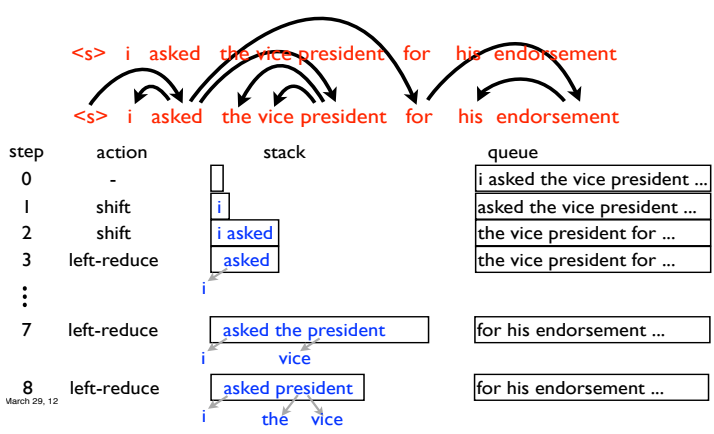

Figure 1: Actions of a shift-reduce parser to produce the dependency structure (up to the word president) shown above.

\section{Dependency Language Models}

Syntactic information can be encoded in terms of headwords and headtags of phrases, which are extracted from a syntactic analysis of a sentence $[1,3]$, such as a dependency structure. A dependency in a sentence holds between a dependent (or modifier) word and a head (or governor) word: the dependent depends on the head. These relations are encoded in a dependency tree (Figure 1), a directed graph where each edge (arc) encodes a head-dependent relation.

We use the shift-reduce incremental dependency parser of [7], which constructs a tree from sequence of transitions governed by a maximum-entropy classifier. Shift-reduce parsing places input words into a queue $Q$ and partially built structures are organized by a stack $S$. Shift and reduce actions consume the queue and build the output parse on the stack. The classifier $g$ assigns probabilities to each action, and the probability of a state $p_{g}(\pi)$ can be computed as the product of the probabilities of a sequence of actions that resulted in the state.

An incremental parser can provide partial syntactic analyses of the history at each word position. Parser states corresponding to the $i$ th word $w_{i}$-states which have $w_{i}$ as the top word in their queue- are history-states, denoted $\Pi_{-i}=$ $\left\{\pi_{-i}^{0}, \pi_{-i}^{1} \cdots, \pi_{-i}^{K_{i}}\right\}$, where $K_{i}$ is the total number of such states (others may be eliminated via beam pruning). Given $\Pi_{-i}$ for all $i$, the probability assignment for $w_{i}$ is:

$p\left(w_{i} \mid W_{-i}\right)=\sum_{j=1}^{\left|\Pi_{-i}\right|} p\left(w_{i} \mid f\left(\pi_{-i}^{j}, W_{-i}\right)\right) p_{g}\left(\pi_{-i}^{j} \mid W_{-i}\right)$

where, $W_{-i}$ is the word history $w_{1}, \ldots, w_{i-1}$ for $w_{i}, \pi_{-i}^{j}$ is the $j^{\text {th }}$ history-state of position $i, p_{g}\left(\pi_{-i}^{j} \mid W_{-i}\right)$ is the probability assigned to state $\pi_{-i}^{j}$ by the parser, and $f\left(\pi_{-i}^{j}, W_{-i}\right)$ denotes an equivalence classification of the word-history and 


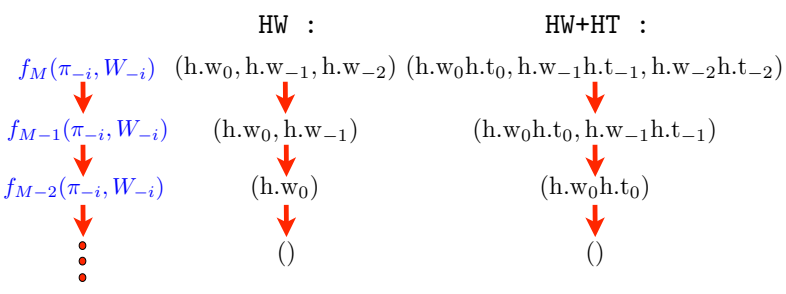

Figure 2: Examples of hierarchal interpolation schemes.

parser history-state, capturing features from $\pi_{-i}^{j}$ and $W_{-i}$ that are most useful for predicting $w_{i}$. We will restrict $f\left(\pi_{-i}^{j}, W_{-i}\right)$ to be based on only the heads of the partial trees $\left\{s_{0} s_{1} \ldots\right\}$ in the stack. For example, in Figure 1, such a function applied to the parser state after step 8 yields the conditional probability $p$ (for|president, NN) for predicting the word for (assuming the top headword president has POS tag NN). Given the choice of $f($.$) , the parameters of the model p\left(w_{i} \mid f\left(\pi_{-i}^{j}, W_{-i}\right)\right)$ are estimated to maximize the log-likelihood of the training data $\mathcal{T}$ using the Baum-Welch algorithm [9].

We use a hiearachy of fine-to-coarse equivalence classifications $f_{M}, f_{M-1}, \ldots, f_{1}$ to smooth probability estimates and handle unseen events via Jelinek-Mercer smoothing [8]. Let

$$
\begin{aligned}
& f_{M}\left(\pi_{-i}, W_{-i}\right) \quad \rightarrow \quad f_{M-1}\left(\pi_{-i}, W_{-i}\right) \quad \rightarrow \quad \ldots \\
& \rightarrow f_{2}\left(\pi_{-i}, W_{-i}\right) \quad \rightarrow \quad f_{1}\left(\pi_{-i}, W_{-i}\right)
\end{aligned}
$$

be a set of $M$ different equivalence classification of history state $\pi_{-i}$. Jelinek-Mercer smoothing utilizes linear interpolation of the ML estimated higher-order equivalence classification probabilities $p_{\mathrm{ML}}\left(w \mid f_{m}\right)$ with lower order $p_{\mathrm{ML}}\left(w \mid f_{m-1}\right)$ :

$$
\begin{aligned}
p_{\text {interp }} & \left(w_{i} \mid f_{m}\left(\pi_{-i} W_{-i}\right)\right) \\
= & \lambda_{f_{m}} p_{\mathrm{ML}}\left(w_{i} \mid f_{m}\left(\pi_{-i} W_{-i}\right)\right) \\
& +\left(1-\lambda_{f_{m}}\right) p_{\text {interp }}\left(w_{i} \mid f_{m-1}\left(\pi_{-i} W_{-i}\right)\right),
\end{aligned}
$$

for $1 \leq m \leq M$, where the 0 -th order model $f_{0}$ is a uniform distribution. Coefficients $\lambda_{f_{m}\left(\pi_{-i} W_{-i}\right)}$ are estimated on a heldout set using the bucketing algorithm suggested in [10], which ties $\lambda_{f_{m}\left(\pi_{-i} W_{-i}\right)}$ 's based on the counts of $f_{m}\left(\pi_{-i} W_{-i}\right)$ 's on the training data. We use the expected count in $\mathcal{T}$ of $\mathbf{f}_{\mathrm{m}}-\mathrm{a}$ specific context feature in the set of all $f_{m}\left(\pi_{-i} W_{-i}\right)$ 's.

We perform the bucketing algorithm for each level $f_{1}, f_{2}, \cdots, f_{M}$ of equivalence classification separately, and estimate the bucketed $\lambda_{c\left(f_{m}\right)}$ using the Baum-Welch algorithm [9] to maximize the likelihood of held out data, where the word probability assignment in Eq. 1 is replaced with:

$p\left(w_{i} \mid W_{-i}\right)=\sum_{j=1}^{\left|\Pi_{i}\right|} p_{\text {interp }}\left(w_{i} \mid f_{M}\left(\pi_{-i}^{j}, W_{-i}\right)\right) p_{g}\left(\pi_{-i}^{j} \mid W_{-i}\right)$

We use two hierarchical interpolation schemes (Figure 2) for smoothing from [1], although we include larger contexts: equivalence classification of history-states by looking only at the headwords (HW) and head-tags $(\mathrm{HW}+\mathrm{HT}$ ) of the first 3 partial trees in the stack. "()" refers to an equivalence classification where no information is used from history (uni-grams.)

\begin{tabular}{|c|c|c|c|c|}
\hline \multicolumn{2}{|c|}{ Language Model } & Eval & Dev & \\
\hline \multicolumn{2}{|c|}{ Kneser-Ney 4-gram } & 158 & 165 & \\
\hline \multirow{3}{*}{ SLM } & $\mathrm{HW}$ & 174 & 183 & \\
\hline & $\mathrm{HW}+\mathrm{HT}$ & 163 & 174 & \\
\hline & $\mathrm{HW}+\mathrm{HT}_{2}$ & 159 & 168 & SLM Weight \\
\hline \multirow{3}{*}{ Interp. } & $\mathrm{HW}$ & 149 & 154 & 0.35 \\
\hline & $\mathrm{HW}+\mathrm{HT}$ & 144 & 150 & 0.43 \\
\hline & $\mathrm{HW}+\mathrm{HT}_{2}$ & 142 & 147 & 0.48 \\
\hline
\end{tabular}

\subsection{Preliminary Preplexity Results}

Before proceeding to our new methods, we establish a baselined perplexity of the SLM using the above two hierarchical schemes. We consider Broadcast News (BN) recognition with
Table 1: The preplexity of different BN language models.

the EARS BN03 corpus, which has $42 M$ words of training text. rt04 (45K words) is used for evaluation data. To interpolate our SLM with the baseline 4-gram model, we use rt03+dev04f (about $40 K$ words) as development data. The vocabulary is $84 K$ words. We sample about $20 K$ sentences from the training text (excluded from training) to serve as heldout data for applying the bucketing algorithm and estimating $\lambda$ 's. For the dependency parser, data sets are converted to Treebank-style tokenization and POS-tagged using the tagger of [11]. Both the POS tagger and dependency parser are trained on the BN treebank from Ontonotes [12] and the WSJ Penn Treebank (converted to dependency trees), which consists of $1.2 \mathrm{M}$ tokens. Finally, we train a modified Kneser-Ney 4-gram LM on the tokenized training text to serve as the baseline LM.

Table 1 shows preplexities obtained for BN . Using headtags in the equivalence classifications used in the HW+HT interpolation scheme significantly improves the preplexity of the SLM. It also improves the performance of the interpolated LM.

\section{Improved Hierarchical Interpolation}

The original SLM hierarchical interpolation scheme is aggressive in that it drops both the tag and headword from the history. However, in many cases the headword's tag alone is sufficient, suggesting a more gradual interpolation. We propose a new interpolation where instead of dropping both the headword and headtag of a partial tree in the stack of a history-state at each level of the HW+HT hierarchical interpolation scheme, we first drop only the headword. E.g., in the HW+HT hierarchy

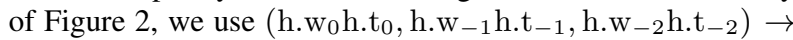

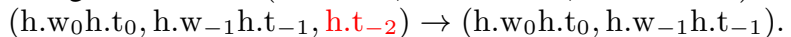

Keeping the headtag adds more specific information and at the same time is less sparse. We refer to this interpolation hierarchy as $\mathrm{HW}+\mathrm{HT}_{2}$. A similar idea is found, e.g., in the backoff hierarchical class $n$-gram language model [13]. Preplexity results (Table 1) confirm that the new scheme improves both interpolated and non-interpolated models.

\section{Relative Entropy Pruning of the SLM}

A significant drawback to the SLM is its size; the space of history-states $\Pi_{-i}$ can be exponentially large. To reduce its size we prune the trained model $p_{\text {interp }}\left(w_{i} \mid f_{M}\left(\pi_{-i}^{j}, W_{-i}\right)\right)$, taking into account accumulated training statistics, as opposed to pruning parser states locally. Our algorithm is based on the pruning scheme proposed in [14], which was used for pruning $n$-gram backoff language models. The goal is to minimize the distance between the implied distributions of the original and pruned models.

The $m^{t h}$-level model in a hierarchical Jelinek-Mercer interpolated LM consists of the following parts:

1. $\mathbf{f}_{\mathbf{m}} \in\left\{f_{m}\right\}$ : The $m^{\text {th }}$-level contextual features which are 


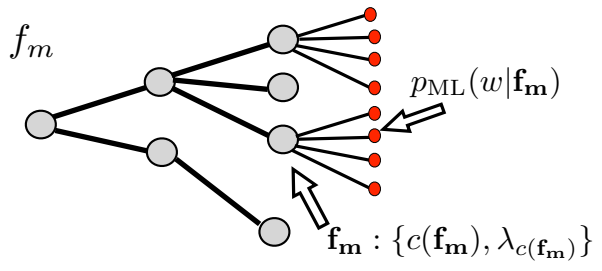

Figure 3: The data structure used for representing $m^{\text {th }}$ level parameters of a Jelinek-Mercer LM.

observed in training.

2. $c\left(\mathbf{f}_{\mathbf{m}}\right)$ : The (empirical expected) count of $\mathbf{f}_{\mathbf{m}}$ in training.

3. $\lambda_{c\left(\mathbf{f}_{\mathbf{m}}\right)}$ : The interpolation weights which are functions of context-feature counts and are used to interpolate $m^{\text {th }}$-level ML probabilities with the $(m-1)^{t h}$-level probabilities.

4. $p_{\mathrm{ML}}\left(w \mid \mathbf{f}_{\mathbf{m}}\right)$ : Explicit maximum-likelihood probability of a word $w$ given the context-feature $\mathbf{f}_{\mathbf{m}}$.

The probability of $w$ given the highest order context-feature $\mathbf{f}_{\mathbf{M}}$ is calculated through the recursive interpolation scheme of Jelinek-Mercer smoothing:

$$
\begin{aligned}
& p_{\text {interp }}\left(w \mid \mathbf{f}_{\mathbf{m}}\right) \\
& \quad=\lambda_{c\left(\mathbf{f}_{\mathbf{m}}\right)} p_{\mathrm{ML}}\left(w \mid \mathbf{f}_{\mathbf{m}}\right)+\left(1-\lambda_{c\left(\mathbf{f}_{\mathbf{m}}\right)}\right) p_{\text {interp }}\left(w \mid \mathbf{f}_{\mathbf{m}-\mathbf{1}}\right),
\end{aligned}
$$

for $1 \leq m \leq M$, where $\mathbf{f}_{\mathrm{M}} \rightarrow \mathbf{f}_{\mathrm{M}-\mathbf{1}} \rightarrow \cdots \rightarrow \mathbf{f}_{\mathbf{1}}$ represents the context-features used in each level of the hierarchical interpolation scheme. Figure 3 depicts the data structure used for representing $m^{t h}$ level parameters of a Jelinek-Mercer LM. The context-features, $\mathbf{f}_{\mathbf{m}}$, and ML probabilities, $p_{\mathrm{ML}}\left(w \mid \mathbf{f}_{\mathbf{m}}\right)$, are efficiently represented using a trie. We refer to pre-leaf and leaf nodes in the trie as "context-nodes" and "probability-nodes", respectively. For a given $m^{t h}$ level context-feature $\mathbf{f}_{\mathbf{m}}$, let

$$
\mathcal{S}\left(\mathbf{f}_{\mathbf{m}}\right)=\left\{w \mid w \in V \text { s.t } p_{\mathrm{ML}}\left(w \mid \mathbf{f}_{\mathbf{m}}\right)>0\right\}
$$

represent all the words in the vocabulary for which there exist an explicit ML estimated probability given the context-feature, i.e. $p_{\mathrm{ML}}\left(w \mid \mathbf{f}_{\mathbf{m}}\right)>0$. In our Jelinek-Mercer pruning scheme, we want to remove those $f_{m} s$ and their corresponding explicit ML probabilities $p_{\mathrm{ML}}\left(w \mid \mathbf{f}_{\mathbf{m}}\right)$ (for $w \in \mathcal{S}\left(\mathbf{f}_{\mathbf{m}}\right)$ ) which have the least impact on the LM distributions. Following [14] we use Kullback-Leibler (KL) divergence to measure the distance between the original and pruned LM. Let $p_{\text {interp }}(. \mid$.) denote the original conditional probabilities and $p_{\text {interp }}^{\prime}(. \mid$.) the pruned ones. The KL divergence using $m^{t h}$ level probabilities is:

$$
D\left(p \| p^{\prime}\right)=\sum_{f_{m}, w} \hat{p}\left(f_{m}\right) p_{\text {interp }}\left(w \mid f_{m}\right) \log \frac{p_{\text {interp }}\left(w \mid f_{m}\right)}{p_{\text {interp }}^{\prime}\left(w \mid f_{m}\right)},
$$

where $\hat{p}\left(f_{m}\right)=\frac{c\left(f_{m}\right)}{\sum_{f_{m}^{\prime}} c\left(f_{m}\right)}$ is the empirical probably of a context-feature $f_{m}$. Ideally, we should minimize Eq. 4 over all possible subsets of $f_{m}$ that are candidates for removal, but this is computationally intractable. Instead, we assume that each $\mathbf{f}_{\mathbf{m}}$ effects $D(\cdot)$ independently and prune those with smallest impact. Removing $\mathbf{f}_{\mathbf{m}}$ changes the model(s) as follows:

(i) For $w \in \mathcal{S}\left(\mathbf{f}_{\mathbf{m}}\right)$, the $m^{\text {th }}$-level probability is completely replaced by the lower-level interpolated model. That is,

$$
\begin{aligned}
p_{\text {interp }}\left(w \mid \mathbf{f}_{\mathbf{m}}\right)= & \lambda_{c\left(\mathbf{f}_{\mathbf{m}}\right)} p_{\mathrm{ML}}\left(w \mid \mathbf{f}_{\mathbf{M}}\right) \\
& +\left(1-\lambda_{c\left(\mathbf{f}_{\mathbf{m}}\right)}\right) p_{\text {interp }}\left(w \mid \mathbf{f}_{\mathbf{m}-\mathbf{1}}\right) \\
p_{\text {interp }}^{\prime}\left(w \mid \mathbf{f}_{\mathbf{m}}\right)= & p_{\text {interp }}\left(w \mid \mathbf{f}_{\mathbf{m}-\mathbf{1}}\right)
\end{aligned}
$$

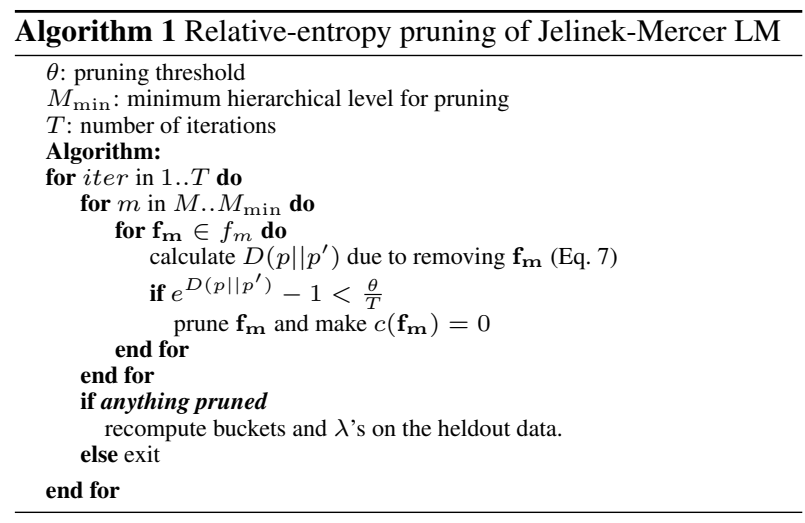

This is because when the context-feature $\mathbf{f}_{\mathbf{m}}$ is removed, it is as if it has not been observed in the training data and $c\left(\mathbf{f}_{\mathbf{m}}\right)$ becomes zero. Therefore, $\lambda_{c\left(\mathbf{f}_{\mathrm{m}}\right)}=0$.

(ii) For $w \notin \mathcal{S}\left(\mathbf{f}_{\mathbf{m}}\right), p_{\mathrm{ML}}\left(w \mid \mathbf{f}_{\mathbf{M}}\right)$ is zero, so that

$$
\begin{aligned}
& p_{\text {interp }}\left(w \mid \mathbf{f}_{\mathbf{m}}\right)=\left(1-\lambda_{c\left(\mathbf{f}_{\mathbf{m}}\right)}\right) p_{\text {interp }}\left(w \mid \mathbf{f}_{\mathbf{m}-\mathbf{1}}\right) \\
& p_{\text {interp }}^{\prime}\left(w \mid \mathbf{f}_{\mathbf{m}}\right)=p_{\text {interp }}\left(w \mid \mathbf{f}_{\mathbf{m}-\mathbf{1}}\right) .
\end{aligned}
$$

(iii) Since we assume $c\left(\mathbf{f}_{\mathbf{m}}\right)=0$ after removing the context-node $\mathbf{f}_{\mathbf{m}}$, the buckets used to tie $\lambda$ 's-and therefore, the maximumlikelihood estimates of $\lambda$ 's on the heldout data-will be affected. This could affect $p_{\text {interp }}^{\prime}\left(w \mid \mathbf{f}_{\mathbf{m}}^{\prime}\right)$ even for $\mathbf{f}_{\mathbf{m}}^{\prime} \neq \mathbf{f}_{\mathbf{m}}$ and is hence computationally difficult to measure. Therefore, we use an iterative algorithm: each iteration measures only the changes in $D\left(p \| p^{\prime}\right)$ due to effects (i) and (ii), ignoring (iii). After pruning $f_{m}$ 's, we run the bucketing algorithm and re-estimate the $\lambda \mathrm{s}$ for the new pruned LM on held out data.

By ignoring (iii) all estimates not involving context-feature $\mathbf{f}_{\mathbf{m}}$ remain unchanged, so the KL divergence from removing $f_{m}$ is:

$$
\begin{array}{r}
D\left(p \| p^{\prime}\right)=p\left(\mathbf{f}_{\mathbf{m}}\right)\left(\sum_{w \in \mathcal{S}\left(\mathbf{f}_{\mathbf{m}}\right)} p_{\text {interp }}\left(w \mid \mathbf{f}_{\mathbf{m}}\right) \log \frac{p_{\text {interp }}\left(w \mid \mathbf{f}_{\mathbf{m}}\right)}{p_{\text {interp }}^{\prime}\left(w \mid \mathbf{f}_{\mathbf{m}}\right)}\right. \\
\left.+\sum_{w \notin \mathcal{S}\left(\mathbf{f}_{\mathbf{m}}\right)} p_{\text {interp }}\left(w \mid \mathbf{f}_{\mathbf{m}}\right) \log \frac{p_{\text {interp }}\left(w \mid \mathbf{f}_{\mathbf{m}}\right)}{p_{\text {interp }}^{\prime}\left(w \mid \mathbf{f}_{\mathbf{m}}\right)}\right)
\end{array}
$$

For the first and second sum we use probabilities in Eq. 5 and Eq. 6, respectively. The above thus simplifies to

$$
\begin{aligned}
& D\left(p \| p^{\prime}\right)=p\left(\mathbf{f}_{\mathbf{m}}\right)\left(\sum_{w \in \mathcal{S}\left(\mathbf{f}_{\mathbf{m}}\right)} p_{\text {interp }}\left(w \mid \mathbf{f}_{\mathbf{m}}\right) \log (A)\right. \\
& \left.+\left[1-\sum_{w \in \mathcal{S}\left(\mathbf{f}_{\mathbf{m}}\right)} p_{\text {interp }}\left(w \mid \mathbf{f}_{\mathbf{m}}\right)\right] \log \left(1-\lambda_{c\left(\mathbf{f}_{\mathbf{m}}\right)}\right)\right)
\end{aligned}
$$

where the term $A$ is,

$$
A=\lambda_{c\left(\mathbf{f}_{\mathbf{m}}\right)} \frac{p_{\mathrm{ML}}\left(w \mid \mathbf{f}_{\mathbf{m}}\right)}{p_{\text {interp }}\left(w \mid \mathbf{f}_{\mathbf{m}-\mathbf{1}}\right)}+\left(1-\lambda_{c\left(\mathbf{f}_{\mathbf{m}}\right)}\right)
$$

Note that a single iteration over all $w \in \mathcal{S}\left(\mathbf{f}_{\mathbf{m}}\right)$ is needed to calculate both the first sum in Eq. 7 and $\sum_{w \in \mathcal{S}\left(\mathbf{f}_{\mathbf{m}}\right)} p_{\text {interp }}\left(w \mid \mathbf{f}_{\mathbf{m}}\right)$.

Algorithm 1 summarizes our proposed pruning procedure. In each iteration, we iterate over all context-features, from level $M$ to $M_{\min }$, and remove nodes for which $\left(e^{D\left(p|| p^{\prime}\right)}-1\right)<\frac{\theta}{T}$. Dividing the threshold $\theta$ by number of iterations reduces pruning in each iteration and distributes pruning across $T$ iterations, where we re-estimate $\lambda$ 's after each iteration to take into account changes due to (iii) above. 


\begin{tabular}{|c|c|c|c|c|}
\hline Threshold & Eval & Dev & \# Param. & Mem. Size \\
\hline \multicolumn{5}{|c|}{ SLM } \\
\hline 0 & 159 & 168 & $413.5 \mathrm{M}$ & $12.5 \mathrm{~GB}$ \\
\hline $1 \times 10^{-7}$ & 159 & 175 & $143.3 \mathrm{M}$ & $4.4 \mathrm{~GB}$ \\
\hline $5 \times 10^{-7}$ & 161 & 183 & $90 \mathrm{M}$ & $2.8 \mathrm{~GB}$ \\
\hline \multicolumn{5}{|c|}{ Interpolated } \\
\hline 0 & 142 & 147 & $(413.5+39) \mathrm{M}$ & $(12.5+1.7) \mathrm{GB}$ \\
\hline $1 \times 10^{-7}$ & 141 & 148 & $(143.3+39) \mathrm{M}$ & $(4.4+1.7) \mathrm{GB}$ \\
\hline $5 \times 10^{-7}$ & 141 & 149 & $(90+39) \mathrm{M}$ & $(2.8+1.7) \mathrm{GB}$ \\
\hline
\end{tabular}

\begin{tabular}{|c|c|c|c|}
\hline \multicolumn{2}{|c|}{ LM } & Pruned & Unpruned \\
\hline \multicolumn{2}{|c|}{ Kneser-Ney 4-gram } & $15.4 \%$ & $15.1 \%$ \\
\hline \multirow{2}{*}{ Interp. SLM } & 1 initial path & $14.7 \%$ & $14.7 \%$ \\
\cline { 2 - 4 } & 20 initial paths & $\mathbf{1 4 . 5} \%$ & $\mathbf{1 4 . 5} \%$ \\
\hline
\end{tabular}

Table 2: Preplexity and memory size of the $\mathrm{HW}+\mathrm{HT}_{2}$ SLMs.

\subsection{Pruning Experiments and Results}

We evaluate the effect of pruning using the setup described in Section 2.1. We apply pruning to our best model, the JelinekMercer SLM with $\mathrm{HW}+\mathrm{HT}_{2}$, with $M=7, T=5$ and $M_{\min }=$ 4. The lowest hierarchy level that we considered for pruning is $\left(\right.$ h. $_{0}$ h. $t_{0}$, h.t $\left._{-1}\right)$. We prune the non-interpolated SLM and then interpolate it with the Kneser-Ney 4-gram model. Our goal is to reduce the SLM to a comparable size to the baseline model, which stores $39 M n$-grams in $1.7 \mathrm{~GB}$ memory.

Pruning reduces the size of the SLMs significantly, as seen in Table 2. SLM size drops from $12.5 \mathrm{~GB}$ to $2.8 \mathrm{~GB}$ without much loss in test-set perplexity. In fact, when interpolated with the Kneser-Ney 4-gram, the Dev-data perplexity is almost unchanged. For the Eval-data, the performance ever so slightly better, possibly due to over-fitting of the unpruned LM.

\section{Speech Recognition Experiments}

We conclude with experiments using our improved SLM for speech recognition. Since the SLM uses equivalence classification of histories based on dependency structures, it is a longspan model, which excludes the ability for direct lattice rescoring. Therefore we use the SLM as the long-span model in the hill-climbing algorithm of [2], which is demonstrably better than $N$-best rescoring.

Since the hill climbing algorithm is a greedy search with no guarantees of global optimality, we use 20 random-restarts, repeating hill climbing with different initial word sequences sampled from the speech lattice, and output the best scoring final output. The hypotheses in the original lattices are not in Treebank-style tokenization, so we tokenize each hypothesis during hill climbing to the Treebank style for POS-tagging, dependency parsing and SLM scoring. The final output is converted back to standard tokenization for WER computation.

We use the SLM with $\mathrm{HW}+\mathrm{HT}_{2}$ interploated with the baseline 4-gram LM. Lattices are generated using a pruned Kneserney 4-gram LM (trained on untokenized training text.) The lattice generating LM has $15.4 \%$ WER and our baseline 4-gram LM (unpruned) has $15.1 \%$ on the evaluation data. We consider both the unpruned and pruned $\left(\theta=5 \times 10^{-7}\right)$ SLM.

The recognition results in Table 3 show that the interpolated SLM significantly improves the baseline performance: a $0.6 \%$ absolute improvement in WER. In addition, our proposed pruning method does not degrade performance, despite significantly reducing LM size. Finally, we observe that hill-climbing with 1 and 20 initial paths results in an average of only 40 and 300 hypotheses evaluated per utterance, a big saving over $N$-best rescoring schemes traditionally employed for long-span language models.

Table 3: Word error rates for speech recognition using the proposed SLM with $\mathrm{HW}+\mathrm{HT}_{2}$ in the hill-climbing algorithm.

\section{Conclusions}

This paper presents useful extensions to the original structured language model to make it more accurate, space efficient and practical for speech recognition. It introduces a simple hierarchical interpolation scheme which improves the preplexity of the SLM. Furthermore, a general information-theoretic pruning algorithm is developed to reduce model size in the JelinekMercer LM smoothing framework. Experiments indicate that this extended SLM framework, when combined with an efficient hill-climbing algorithm, improves recognition accuracy over regular $n$-gram models.

\section{References}

[1] C. Chelba and F. Jelinek, "Structured language modeling," Computer Speech and Language, vol. 14, no. 4, pp. 283-332, 2000.

[2] A. Rastrow, M. Dreyer, A. Sethy, S. Khudanpur, B. Ramabhadran, and M. Dredze, "Hill climbing on speech lattices : A new rescoring framework," in Proceeding of ICASSP, 2011.

[3] H. K. J. Kuo, L. Mangu, A. Emami, I. Zitouni, and L. YoungSuk, "Syntactic features for Arabic speech recognition," in Proc. ASRU, 2009.

[4] M. Collins, B. Roark, and M. Saraclar, "Discriminative syntactic language modeling for speech recognition," in ACL, 2005.

[5] S. Khudanpur and J. Wu, "Maximum entropy techniques for exploiting syntactic, semantic and collocational dependencies in language modeling," Computer Speech and Language, pp. 355-372, 2000.

[6] A. Rastrow, M. Dredze, and S. Khudanpur, "Efficient discrimnative training of long-span language models," in IEEE Workshop on Automatic Speech Recognition and Understanding (ASRU), 2011.

[7] K. Sagae and J. Tsujii, "Dependency parsing and domain adaptation with LR models and parser ensembles," in Proc. EMNLPCoNLL, vol. 7, 2007, pp. 1044-1050.

[8] F. Jelinek and R. L. Mercer, "Interpolated estimation of Markov source parameters from sparse data," in Proceedings of the Workshop on Pattern Recognition in Practice, 1980, pp. 381-397.

[9] L. E. Baum, "An equality and associated maximization technique in statistical estimation for probabilistic functions of Markov processes," Inequalities, vol. 3, pp. 1-8, 1972.

[10] L. Bahl, "A maximum likelihood approach to continuous speech recognition," IEEE Transactions on Pattern Analysis and Machine Inteligence (PAMI), vol. 5, no. 2, pp. 179-190, 1983.

[11] Y. Tsuruoka, Y. Miyao, and J. Kazama, "Learning with Lookahead : Can History-Based Models Rival Globally Optimized Models?"' in Proc. CoNLL, no. June, 2011, pp. 238-246.

[12] R. Weischedel, S. Pradhan, L. Ramshaw, M. Palmer, N. Xue, M. Marcus, A. Taylor, C. Greenberg, E. Hovy, R. Belvin, and A. Houston, OntoNotes Release 2.0, Linguistic Data Consortium, Philadelphia, 2008.

[13] I. Zitouni, "Backoff hierarchical class n-gram language models: effectiveness to model unseen events in speech recognition," Computer Speech \& Language, vol. 21, no. 1, pp. 88-104, 2007.

[14] A. Stolcke, "Entropy-based pruning of backoff language models," in Proc. DARPA Broadcast News Transcription and Understanding Workshop, 1998. 